\title{
A Multilead Scheme Based on Periodic Component Analysis for T-Wave Alternans Analysis in the ECG
}

\author{
Violeta Monasterio, ${ }^{1,2}$ Gari D. Clifford, ${ }^{3}$ Pablo Laguna, ${ }^{1,2}$ and Juan Pablo Martínez ${ }^{1,2}$ \\ ${ }^{1}$ CIBER de Bioingeniería, Biomateriales y Nanomedicina (CIBER-BBN), Zaragoza, Spain; ${ }^{2}$ Communications Technology \\ Group, Aragon Institute of Engineering Research, Universidad de Zaragoza, Zaragoza, Spain; and ${ }^{3}$ Department of Engineering \\ Science, Institute of Biomedical Engineering, University of Oxford, Oxford, UK
}

(Received 20 January 2010; accepted 27 March 2010; published online 13 April 2010)

Associate Editor Leonidas D. Iasemidis oversaw the review of this article.

\begin{abstract}
T-wave alternans (TWA) is a cardiac phenomenon that appears in the electrocardiogram (ECG) and is associated with the mechanisms leading to sudden cardiac death (SCD). In this study, we propose the use of a multilead TWA analysis scheme that combines the Laplacian likelihood ratio (LLR) method and periodic component analysis ( $\pi \mathrm{CA})$, an eigenvalue decomposition technique whose aim is to extract the most periodic sources of the signal. The proposed scheme is evaluated in different scenarios-from synthetic signals to stress test ECGs - and is compared to other reported schemes based on the LLR method. Results demonstrate that the $\pi$ CA-based scheme provides a superior ability to detect TWA than previously reported schemes, and has the potential to improve the prognostic value of testing for TWA.
\end{abstract}

Keywords-Electrocardiogram, T-wave alternans, Multilead analysis, Periodic component analysis.

\section{INTRODUCTION}

T-wave alternans (TWA) is a phenomenon appearing in the electrocardiogram (ECG) as a consistent fluctuation in the morphology of the ventricular repolarization on an every-other-beat basis, and is currently regarded as a promising index of susceptibility to sudden cardiac death (SCD).$^{19,23}$ TWA has been shown to reflect the presence of abnormal repolarization and electrophysiologic inhomogeneities that underlie vulnerability to ventricular fibrillation. ${ }^{19}$ Measurement of TWA during controlled heart rate elevation is considered as a potentially useful predictor of ventricular arrhythmic events and mortality in

Address correspondence to Violeta Monasterio, Communications Technology Group, Aragon Institute of Engineering Research, Universidad de Zaragoza, Zaragoza, Spain. Electronic mail: violeta@ unizar.es patients with ischemic cardiomyopathy, ${ }^{3}$ and its role in identification of patients that would benefit ICD implantation is being currently studied. ${ }^{6}$

TWA amplitudes are in the range of microvolts, and can be even below the noise level, making the detection of TWA a difficult task. Several methods exist to automatically detect and estimate TWA. ${ }^{12}$ Two commercially available techniques are the spectral method $(\mathrm{SM})^{23,26}$ and the modified moving average method $^{22}$; alternative techniques are the complex demodulation method $^{20}$ and the Laplacian likelihood ratio method (LLR). ${ }^{11,13}$ A major limitation of these methods is their poor sensitivity to low-amplitude alternans ${ }^{12,19}$ due to a lack of robustness to noise. With SM, for example, a noise level $\geq 2 \mu \mathrm{V}$ in a predefined spectral band can lead to an indeterminate TWA test. ${ }^{2}$

Existing methods are mostly applied to each ECG lead individually; only basic multilead strategies, such as analyzing the vector-magnitude lead, are applied in commercial systems (CH2000 and Heartwave systems, Cambridge Heart Inc, Bedford, MA). In a previous work, ${ }^{16}$ we presented a multilead scheme based on principal component analysis (PCA) that improved the estimation and detection performance of SM and LLR single-lead techniques. This improvement, however, was limited by the fact that PCA uses a maximumvariance criterion to separate signal and noise into orthogonal subspaces, which may not be the best way of differentiating between the constituent sources of an ECG signal, particularly if they are not orthogonal. ${ }^{4}$

In this study, we present a new multilead scheme based on periodic component analysis $(\pi \mathrm{CA})$, a technique that was first proposed in Saul and Allen ${ }^{25}$ and later applied to ECG signals in Sameni et al. ${ }^{24}$ With this technique, the variance criterion of PCA is replaced by a periodical structure criterion to separate 
TWA from non-alternant components, which is a more reasonable assumption from a physiological point of view because periodicity is the defining characteristic of TWA. In this study, two multilead schemes, one based on $\pi \mathrm{CA}$ and the other based on PCA, are combined with the LLR method, and are compared to a conventional single-lead approach, in which each ECG lead is analyzed independently. Results on simulated signals with different types of noise, real signals with added TWA, and stress test signals, show that the $\pi$ CA-based scheme provides the best detection power and has the potential to improve the prognostic value of TWA tests.

\section{METHODS FOR TWA ANALYSIS}

Three different approaches are compared: a multilead scheme based on $\pi \mathrm{CA}$ (multi- $\pi C A$ ), a multilead scheme based on PCA (multi-PCA), ${ }^{16}$ and a single-lead scheme (single).

\section{General Multilead Scheme}

Both multi-PCA and multi- $\pi C A$ approaches follow a general scheme that consists of five stages: signal preprocessing, signal transformation, TWA detection, signal reconstruction, and TWA estimation (Fig. 1). The difference between multi-PCA and multi- $\pi C A$ is the way in which the transformation matrix is obtained.

\section{Signal Preprocessing}

QRS positions are determined using a wavelet-based algorithm. ${ }^{10}$ Baseline wander is removed with a cubic splines interpolation technique. ${ }^{14}$ The frequency content of TWA is located below $15 \mathrm{~Hz},{ }^{21}$ so the ECG signal can be low-pass filtered and downsampled to a new sampling frequency $F_{\mathrm{s}} \geq 30 \mathrm{~Hz}$ to remove offband noise. In this study, signals with sampling frequencies of $1 \mathrm{kHz}$ and $500 \mathrm{~Hz}$ are decimated by a factor of 32 and 16, respectively, obtaining a
$F_{\mathrm{s}}=31.25 \mathrm{~Hz}$. In the case of 12-lead ECGs, only the eight independent leads (V1-V6, I, II) are considered. Figure 2a shows an example of a real signal where TWA of $200 \mu \mathrm{V}$ was artificially added. This signal belongs to the dataset described in section "Real Signals with Added TWA".

In each beat, an interval of $350 \mathrm{~ms}$ - or the closest value corresponding to a integer number of samples - is selected for TWA analysis after the end of the QRS (ST-T complex). An interval of $352 \mathrm{~ms}$ (11 samples) was selected in this study. Let $K$ be the number of beats in the analysis window, $N$ the number of samples of each ST-T complex, $L$ the number of leads, and $x_{k, l}(n)$ the $n$th sample in the ST-T complex of the $k$ th beat and the $l$ th lead. Each ST-T complex can be modeled as

$$
\begin{gathered}
x_{k, l}(n)=s_{l}(n)+\frac{1}{2} a_{l}(n)(-1)^{k}+v_{k, l}(n), \\
n=0, \ldots, N-1,
\end{gathered}
$$

where $s_{l}(n)$ is the background ST-T complex, which is periodically repeated in each beat, $a_{l}(n)$ is the alternans waveform, and $v_{k, l}(n)$ is additive random noise. In vector notation, each ST-T complex is denoted as

$$
\mathbf{x}_{k, l}=\left[x_{k, l}(0) \ldots x_{k, l}(N-1)\right]^{T} .
$$

For each beat $k$, complexes from all leads are put together into a matrix $\mathbf{X}_{k}$ :

$$
\mathbf{X}_{k}=\left[\mathbf{x}_{k, 1} \ldots \mathbf{x}_{k, L}\right]^{T}
$$

The $n$th column of $\mathbf{X}_{k}$ contains the amplitudes of the $L$ leads at a given sample $n$ of the $k$ th beat. A data matrix $\mathbb{X}$ is then constructed by concatenating the $\mathbf{X}_{k}$,

$$
\mathbb{X}=\left[\mathbf{X}_{0} \mathbf{X}_{1} \ldots \mathbf{X}_{K-1}\right]
$$

and finally a matrix $\mathbb{X}^{(\mathrm{m})}$ is constructed as

$$
\mathbb{X}^{(m)}=\left[\mathbf{X}_{m} \mathbf{X}_{m+1} \ldots \mathbf{X}_{m+K-1}\right]
$$

that can be interpreted as the equivalent of $\mathbb{X}$, but obtained after sliding the analysis window $m$ beats forward.

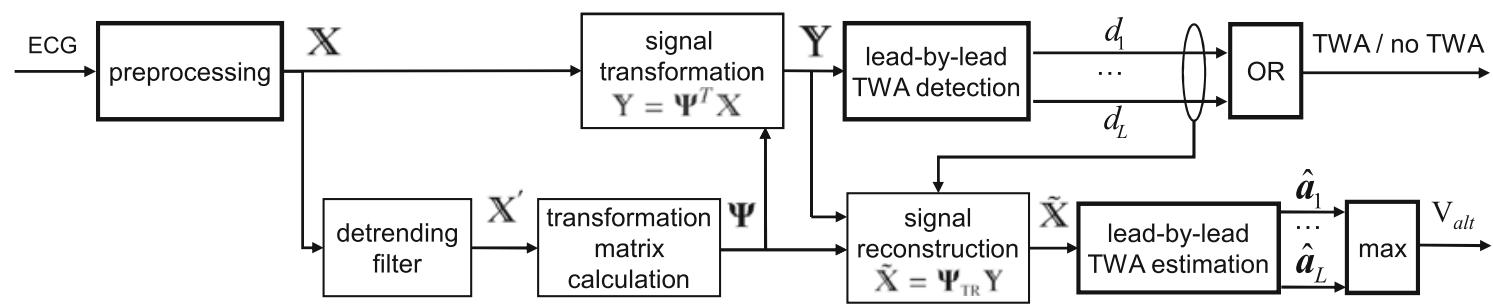

FIGURE 1. Block diagram of the general TWA multilead analysis scheme. Blocks in bold line are the ones used in the single-lead scheme, in which $Y=X=\tilde{X}$. 


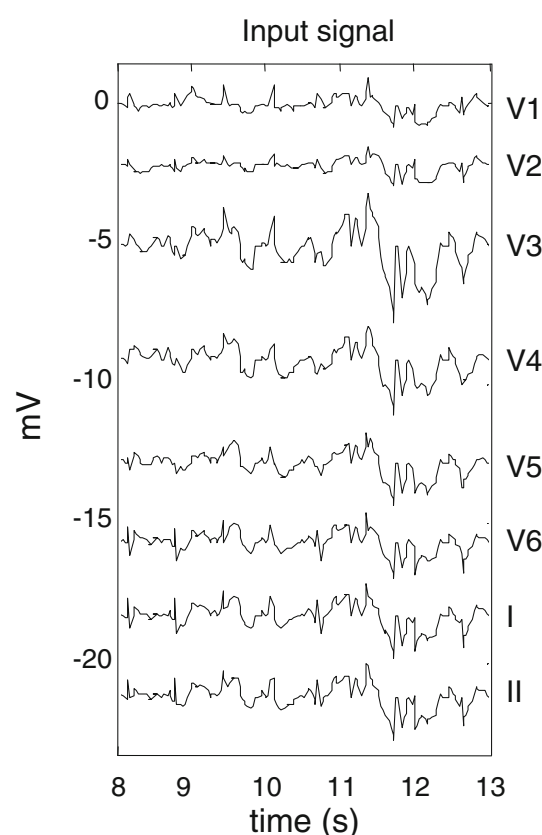

(a)

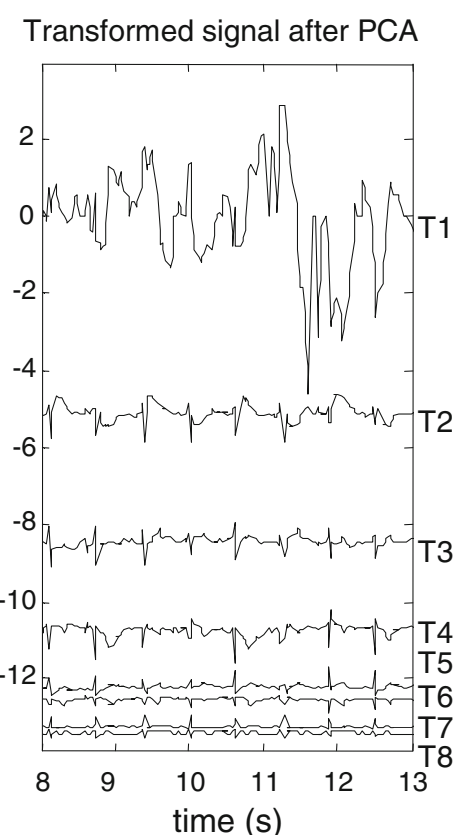

(b)
Transformed signal after $\pi \mathrm{CA}$

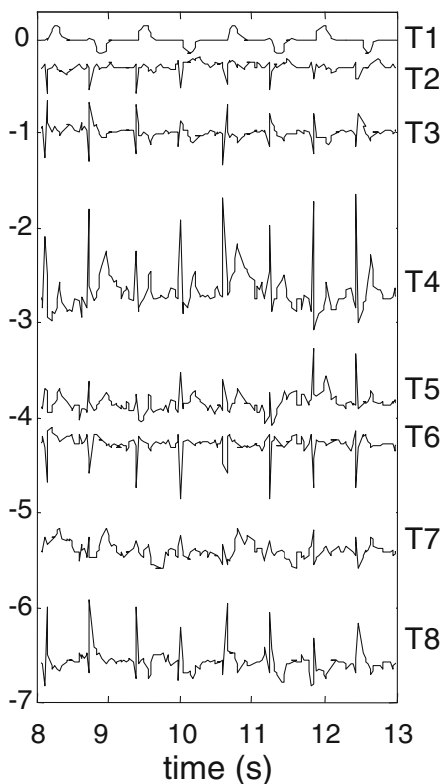

(c)

FIGURE 2. (a) Eight independent leads of a real 12-lead ECG where TWA of $200 \mu \mathrm{V}$ was artificially added. TWA is invisible to the naked eye due to noise and artifacts. (b) Signal in (a) after PCA transformation. TWA is now visible in T2 through exaggerated oscillations in the amplitude of the T wave. (c) Signal in (a) after $\pi$ CA transformation. TWA is clearly visible in T1.

\section{Signal Transformation}

The objective of this stage is to find a linear transformation $\mathbb{Y}=\Psi^{T} \mathbb{X}$ that improves the signal-to-noise ratio (SNR) of TWA by exploiting spatial and temporal information of the multilead ECG. The leads of the transformed signal $Y$ will be denoted as transformed leads from here on $(\mathrm{T} l, l=1 \ldots L)$. First, background ST-T complexes are canceled with a detrending filter that computes the difference between each complex and the previous one:

$$
\mathbf{x}_{k, l}^{\prime}=\mathbf{x}_{k, l}-\mathbf{x}_{k-1, l}, \quad k=1 \ldots K-1
$$

and the detrended beats $\mathbf{x}_{k, l}^{\prime}$ are arranged as in (2) and (3) to form matrices $\mathbb{X}^{\prime}$ and $\mathbb{X}^{(m)^{\prime}}$, respectively. Note that $\mathbb{X}^{\prime}$ and $\mathbb{X}^{(m)^{\prime}}$ now contain $K-1$ beats. Transformation $\boldsymbol{\Psi}$ is then calculated with PCA or with $\pi \mathrm{CA}$.

\section{Principal Component Analysis}

The detrended signal $\mathbb{X}^{\prime}$ is a zero-mean random process with a spatial correlation that can be estimated as

$$
\mathbf{R}_{\mathbb{X}^{\prime}}=\frac{1}{(K-1) N} \mathbb{X}^{\prime} \mathbb{X}^{\prime T}
$$

To obtain the required transformation, the eigenvector equation for $\mathbf{R}_{\mathbb{X}^{\prime}}$ is solved:

$$
\mathbf{R}_{\mathbb{X}^{\prime}} \boldsymbol{\Psi}=\boldsymbol{\Psi} \Lambda,
$$

where $\boldsymbol{\Lambda}$ denotes the eigenvalue matrix with the eigenvalues sorted in descending order, and $\Psi$ is the corresponding eigenvector matrix. The matrix $\boldsymbol{\Psi}$ defines an orthonormal transformation, which is applied to the original data $\mathbb{X}$

$$
\mathbb{Y}=\boldsymbol{\Psi}^{\mathbb{T}} \mathbb{X}
$$

to obtain the transformed signal, whose $l$ th transformed lead (lth row in $\mathbb{Y}$ ) contains the $l$ th principal component of $\mathbb{X}$. Figure $2 \mathrm{~b}$ shows the input signal in (a) after PCA transformation.

\section{Periodic Component Analysis}

This technique aims to find the transformation $\mathbf{y}_{k}^{\prime T}=\mathbf{w}^{T} \mathbb{X}_{k}^{\prime}$ that maximizes the periodic structure of the signal at the TWA frequency, which is 0.5 cycles per beat (cpb), or equivalently at period $m=2$ beats. The desired transformation must minimize the following measure of periodicity:

$$
\epsilon(\mathbf{w}, m)=\frac{\sum_{k=0}^{K-1}\left\|\mathbf{y}_{k+m}^{\prime}-\mathbf{y}_{k}^{\prime}\right\|^{2}}{\sum_{k=0}^{K-1}\left\|\mathbf{y}_{k}^{\prime}\right\|^{2}}
$$

As shown in Sameni et al., ${ }^{24}$ Eq. (8) can be rearranged as

$$
\epsilon(\mathbf{w}, m)=\frac{\mathbf{w}^{T} \mathbf{A}_{\mathbb{X}^{\prime}}(m) \mathbf{w}}{\mathbf{w}^{T} \mathbf{R}_{\mathbb{X}^{\prime}} \mathbf{w}},
$$

where $\mathbf{R}_{\mathbb{X}^{\prime}}$ is defined in (5) and $\mathbf{A}_{\mathbb{X}^{\prime}}(m)$ is the spatial correlation of $\left(\mathbb{X}^{(m)^{\prime}}-\mathbb{X}^{\prime}\right)$, which can be estimated as

$$
\mathbf{A}_{\mathbb{X}^{\prime}}(m)=\frac{1}{(K-1) N}\left(\mathbb{X}^{(m)^{\prime}}-\mathbb{X}^{\prime}\right)\left(\mathbb{X}^{(m o n) \prime}-\mathbb{X}^{\prime}\right)^{T} .
$$


The weight $\mathbf{w}$ that minimizes (9) is given by the generalized eigenvector corresponding to the smallest generalized eigenvalue of the matrix pair $\left(\mathbf{A}_{\mathbb{X}^{\prime}}(m), \mathbf{R}_{\mathbb{X}^{\prime}}\right) .{ }^{24,25}$ The transformation matrix $\boldsymbol{\Psi}$ is chosen as the generalized eigenvector matrix of $\left(\mathbf{A}_{\mathbb{X}^{\prime}}(m), \mathbf{R}_{\mathbb{X}^{\prime}}\right)$, with the eigenvectors (columns) sorted according to the corresponding eigenvalues in ascending order of magnitude. In this way, the transformation $\mathbb{Y}=\Psi^{T} \mathbb{X}$ projects the most periodic component into the first row of $\mathbb{Y}$, i.e., TWA, if present, is projected into the first transformed lead. Figure 2c illustrates the input signal in (a) after $\pi \mathrm{CA}$ transformation.

\section{TWA Detection}

After signal transformation, TWA detection is performed in the transformed signal $\mathbb{Y}$ using the LLR method. ${ }^{11,13}$ This method applies the generalized likelihood ratio test (GLRT) for Laplacian noise to each transformed lead (rows in $\mathbb{Y}$ ) as follows. A detection statistic $Z$, which represents an indirect measure of the quotient between alternans and noise power (the alternans-to-noise ratio or ANR), is computed from the data. This statistic $Z$ is compared to a threshold $\gamma$ to decide whether alternans is present $(Z \geq \gamma)$ or not, and the result of this lead-by-lead detection is denoted as $d_{l}=1$ if TWA is present in the $l$ th transformed lead, and $d_{l}=0$ otherwise. The overall TWA detection is positive if $Z \geq \gamma$ at least in one transformed lead ('OR' block in Fig. 1), and negative otherwise.

\section{Signal Reconstruction}

TWA must be measured in the original leads to be clinically useful. Therefore, a new signal in the original lead set is reconstructed, considering only the transformed leads where TWA was detected. The matrix $\Psi$ is modified by inserting zeros in the columns corresponding to leads without TWA, thus obtaining a matrix $\boldsymbol{\Psi}_{\mathrm{TR}}$, and then the reconstructed signal is obtained as

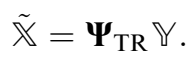

\section{TWA Estimation}

The maximum likelihood estimation (MLE) for Laplacian noise is applied to each reconstructed lead $l$ as described in Martínez et al. ${ }^{13}$ to estimate the TWA waveform, $\hat{a}_{l}(n)$. The global amplitude of TWA $\left(V_{\text {alt }}\right)$ is finally defined as

$$
V_{\mathrm{alt}}=\max \left\{\sqrt{\frac{1}{N} \sum_{n=0}^{N-1} \hat{a}_{l}^{2}(n)}\right\}_{l=1 \ldots L}(\mu V) .
$$

\section{Single-Lead Scheme}

The single-lead scheme handles each lead independently throughout the process. It consists of the same preprocessing, TWA estimation, and TWA detection stages as the multilead scheme, but without the intermediate transformation/reconstruction stages. That is, with the single-lead scheme $\mathbb{Y}=\mathbb{X}=\tilde{\mathbb{X}}$. The stages of the single-lead scheme are shown as bold line in Fig. 1.

\section{DATA SETS}

To compare the analysis schemes in a variety of scenarios, three data sets with increasing degree of realism were analyzed. The first data set was composed of synthetic ECG signals with simulated TWA. In the second data set, known TWA was added to real ECG signals. The third data set contained real stress test ECGs from healthy volunteers and ischemic patients. Both second and third sets were composed of ECG records from a stress test database recorded in the University Hospital Lozano Blesa of Zaragoza (Spain). ${ }^{1}$ This database contains 12-lead ECGs recorded during treadmill exercise test following Bruce protocol at $1 \mathrm{kHz}$ sampling rate with an amplitude resolution of $0.6 \mu \mathrm{V}$. Records belong to three different groups: 211 patients with a negative clinical and electrical exercise test (non-ischemic group), 66 asymptomatic volunteers from the Spanish Army who underwent the exercise test with negative results for coronary artery disease (volunteer group), and 79 patients with significant stenosis in at least one major coronary artery as shown by coronary angiography (ischemic group).

Also, a publicly available dataset was analyzed to facilitate future reproduction or comparison with the methods presented in this article.

\section{Synthetic Signals}

Synthetic 3 Frank lead ECGs were generated using an artificial multilead ECG model which can exhibit realistic TWA. ${ }^{5}$ This model generates the cardiac dipole as a sum of Gaussian kernels, and then derives the XYZ orthogonal leads. Natural morphology changes due to inter-beat interval (RR), and respiration are incorporated from varying the Gaussian amplitude and width parameters of the dipole generator. The TWA effect is modeled as a disturbance on the T-loop of the dipole.

Five-minute signals were created with TWA amplitudes ranging from 0 to $136 \mu \mathrm{V}$. For a given TWA amplitude, a total of $50 \mathrm{ECG}$ records were generated at a sampling frequency of $500 \mathrm{~Hz}$. Individual records differed in that the underlying ECGs were generated 
using an stochastic model of heart rate variability $(70 \pm 5$ beats per minute $(\mathrm{bpm}))$. Furthermore, a mixture of noises - electrode motion (em), muscular activity $(m a)$, and baseline wander $(b w)$, obtained from the MIT-BIH Noise Stress Test Database ${ }^{18}$ —was added to the signals. For each synthetic ECG signal, two-lead segments of 5 min were extracted from em, $m a$, and $b w$ records beginning at a random position. These records only contain two leads, so a third lead of spatially correlated noise was computed as the first principal component of the two available leads. Finally, the three noise leads were scaled to obtain an SNR of $20 \mathrm{~dB}$ with respect to the ECG, and added to the synthetic signal.

\section{Real Signals with Added TWA}

This data set contained real 12-lead ECGs with artificially added TWA. ECG fragments where the presence of TWA was unlikely were selected from all non-ischemic and volunteer records. The TWA phenomenon is partially related to heart rate, and can appear in healthy subjects at fast heart rates. ${ }^{7,27}$ Therefore, only the initial fragment in which the heart rhythm was $<100 \mathrm{bpm}$ was selected from each record. These fragments were then divided into segments of 32 consecutive beats, resulting in a total of 1744 ECG segments. Then, TWA was added to ECGs, with amplitudes $\left(V_{\text {alt }}\right)$ ranging from 0 to $200 \mu \mathrm{V}$. To do this, the LLR method was employed to identify a real 12-lead TWA waveform in an ECG of a patient undergoing a percutaneous coronary intervention, a procedure that can trigger TWA due to the resultant severe ischemia. ${ }^{13}$ For each $V_{\text {alt }}$ value, the TWA waveform was scaled and repeated with alternating sign to create a 32-beat-long TWA signal, and then the TWA signal was added to the 1744 ECG segments. This process was repeated with two more 12-lead TWA waveforms extracted from two different records of the same database. ${ }^{13}$

\section{Stress Test Signals}

The third data set comprised all ECGs from ischemic and volunteer groups of the stress test database. Volunteer records had an average duration of $20 \mathrm{~min}$, and the maximum heart rate over the test was $183 \pm 8 \mathrm{bpm}$ (mean \pm standard deviation). Ischemic records had an average duration of $16 \mathrm{~min}$, and the maximum heart rate over the test was $134 \pm$ $23 \mathrm{bpm}$.

\section{Signals from the Physionet TWA Database}

The Physionet TWA database is a publicly available resource compiled for the PhysioNet/Computers in
Cardiology Challenge 2008. ${ }^{17}$ It contains 100 real and synthetic ECG records, sampled at $500 \mathrm{~Hz}$ and with an approximate duration of $2 \mathrm{~min}$. The synthetic subset of the database consists of thirty-two 12-lead ECGs containing artificial TWA in calibrated amounts.

\section{RESULTS}

\section{Synthetic Signals}

Signals were processed with the single-lead and the PCA and $\pi \mathrm{CA}$ multilead schemes using a 32-beat analysis window. Following the terminology of detection theory, probability of false alarm $\left(P_{\mathrm{FA}}\right)$ was defined as the ratio between the number of positive TWA detections in signals with $V_{\text {alt }}=0$ (false detections) and the total number of decisions made in those signals. Probability of detection $\left(P_{\mathrm{D}}\right)$ was defined as the ratio between the number of positive TWA detections in signals with a given $V_{\text {alt }}$ and the total number of decisions made in those signals. For each scheme, the detection threshold $\gamma$ was set so that the probability of false alarm $\left(P_{\mathrm{FA}}\right)$ was 0.01 , and the resulting $P_{\mathrm{D}}$ was compared. In the range $30 \mu \mathrm{V} \leq V_{\text {alt }} \leq 100 \mu \mathrm{V}$, the multi- $\pi C A$ scheme detected alternans with amplitudes at least $15 \mu \mathrm{V}$ lower than the single scheme for a given $P_{\mathrm{D}}$ (Fig. 3).

The estimation accuracy of the schemes was also compared. For each simulated amplitude, the mean value and the standard deviation of the estimated amplitude $V_{\text {alt }}$ were computed considering only those cases where detection was positive (Fig. 4). In absence of TWA, TWA was falsely detected in $1 \%$ of the cases

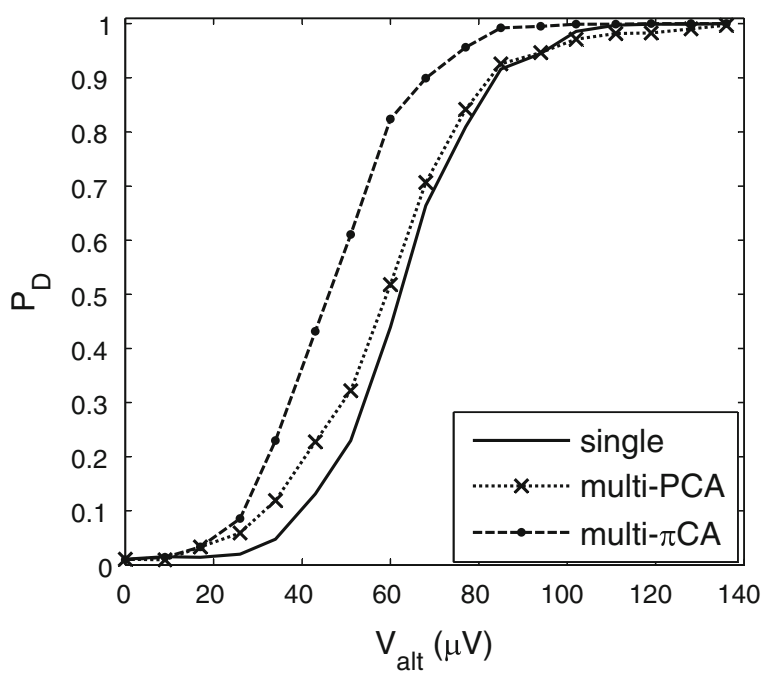

FIGURE 3. $P_{\mathrm{D}}$ of multi-PCA, multi- $\pi C A$, and single schemes obtained in synthetic signals vs. simulated TWA amplitude. $P_{\mathrm{FA}}=\mathbf{0 . 0 1}$ for all schemes. 

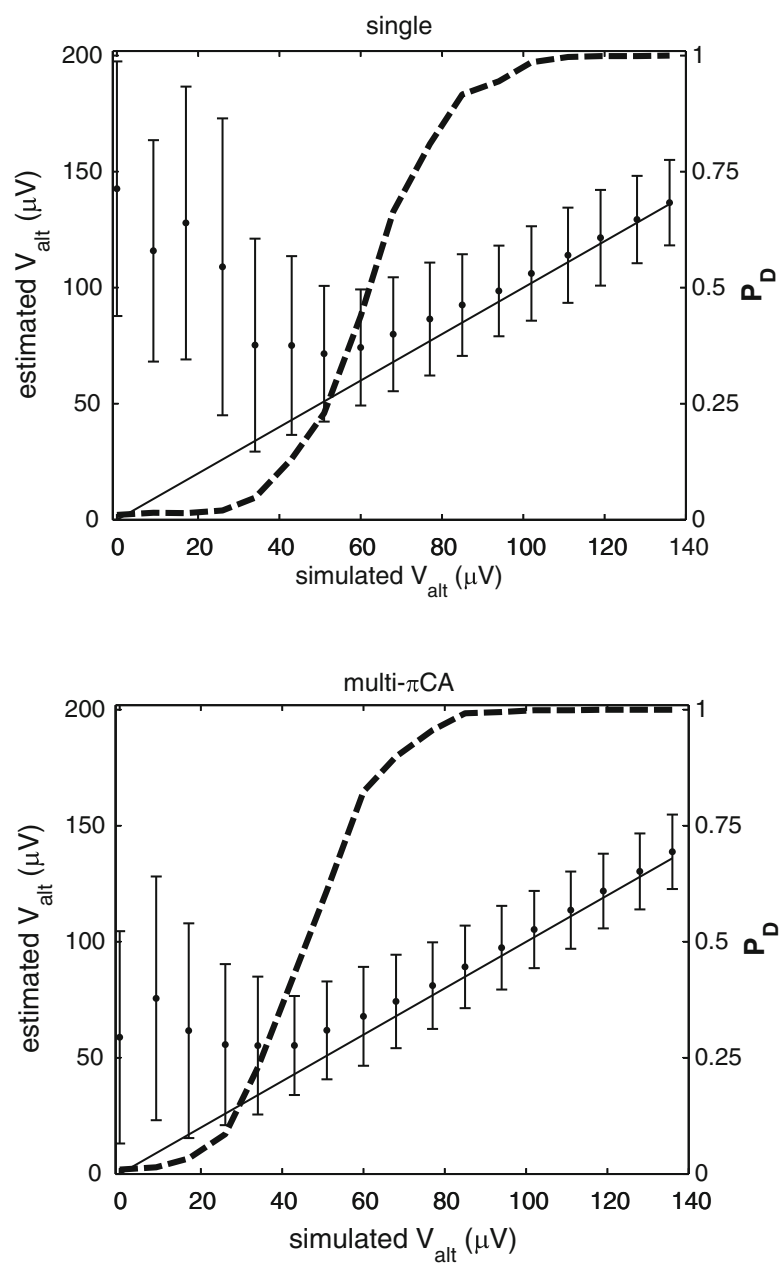

FIGURE 4. Estimated vs. simulated TWA amplitude in synthetic signals using the single-lead (top), and multi- $\pi C A$ (bottom) schemes. Dots and bars represent the average and the standard deviation of the estimated amplitude respectively, and straight line corresponds to perfect estimation (left ordinates axis). Dashed line represents the $P_{\mathrm{D}}$ of each scheme for $P_{\mathrm{FA}}=0.01$ (right ordinates axis).

with a mean estimated amplitude of $142.6 \mu \mathrm{V}$ (single scheme) and $58.9 \mu \mathrm{V}$ (multi- $\pi C A$ scheme). For a simulated amplitude of $136 \mu \mathrm{V}$, the bias and standard deviation of the estimation were 0.6 and $18.2 \mu \mathrm{V}$, respectively (for single scheme), and 2.7 and $16.0 \mu \mathrm{V}$ (for multi- $\pi C A$ scheme).

\section{Real Signals with Added TWA}

Real signals were processed in the same way as synthetic signals. For each scheme, the detection threshold $\gamma$ was set so that $P_{\mathrm{FA}}=0.01$, and the resulting $P_{\mathrm{D}}$ was compared (Fig. 5). TWA of $5 \mu \mathrm{V}$ was detected with a $P_{\mathrm{D}}=1 \%$ by the single scheme, with a $P_{\mathrm{D}}=60 \%$ by the multi-PCA scheme, and with a $P_{\mathrm{D}}=98 \%$ by the multi- $\pi C A$ scheme. Similar detection

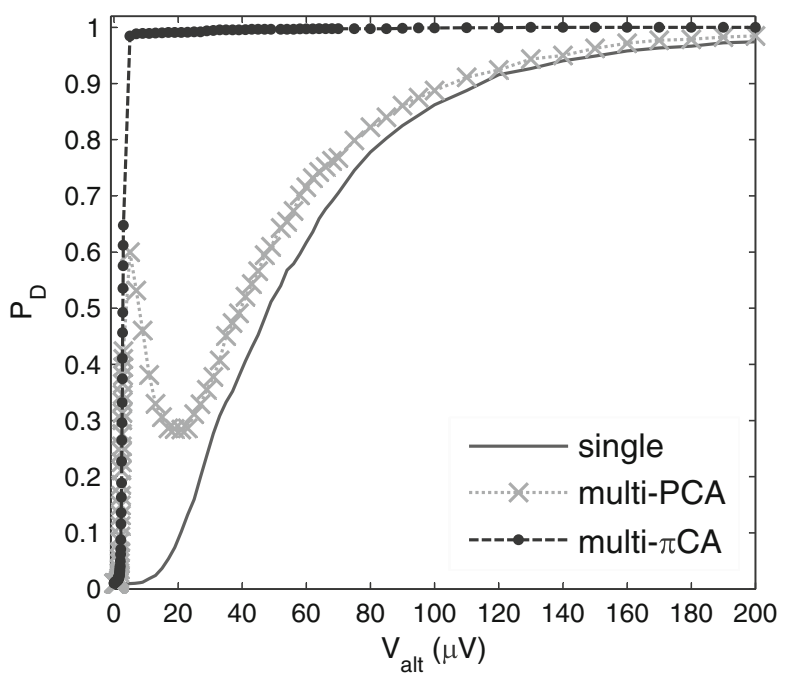

FIGURE 5. $P_{\mathrm{D}}$ in real signals with added TWA with multi$P C A$, multi- $\pi C A$, and single schemes vs. TWA amplitude. $P_{\mathrm{FA}}=\mathbf{0 . 0 1}$ for all schemes.

differences were found by repeating the analysis with two other TWA waveforms. The triphasic behavior of the multi-PCA $P_{\mathrm{D}}$ curve was also found in all cases.

To better understand the effects of PCA and $\pi \mathrm{CA}$ transformations, the evolution of the GLRT statistic $Z$ in the transformed leads vs. TWA amplitude was analyzed for all signals. Figure 6 shows an example where $Z$ is computed on an ECG signal without any transformation (top panel), after PCA transformation (middle panel), or after $\pi \mathrm{CA}$ transformation (bottom panel). In all cases, TWA is detected when $Z$ exceeds the threshold (horizontal black line) in one or more leads. With PCA, TWA was detected for $V_{\text {alt }}<5 \mu \mathrm{V}$, become undetected for $5<V_{\text {alt }}<50 \mu \mathrm{V}$, and then was detected again in $\mathrm{T} 2$ for $V_{\text {alt }}=50 \mu \mathrm{V}$. With $\pi \mathrm{CA}$, TWA was detected for $V_{\text {alt }} \geq 3.5 \mu \mathrm{V}$.

\section{Stress Test Signals}

Stress test records were processed with multi- $\pi C A$ and single schemes. Comparison between multi-PCA and single-lead schemes had already been reported in Monasterio et al. ${ }^{16}$ and therefore the multi-PCA scheme was excluded from this study.

From the 12-lead set, only the eight independent leads were processed using a sliding analysis window of 128 beats. This window length was chosen because it is commonly used in commercial equipments. To make a fair comparison of the sensitivity of multi- $\pi C A$ and single schemes, their specificity was made equal by setting the same $P_{\mathrm{FA}}=0.01$ for both schemes. To do so, the assumption that no TWA is likely to be found in volunteer records at heart rates below a cut-off value $H R_{\mathrm{c}}$ was made. Volunteers' signals were processed, 

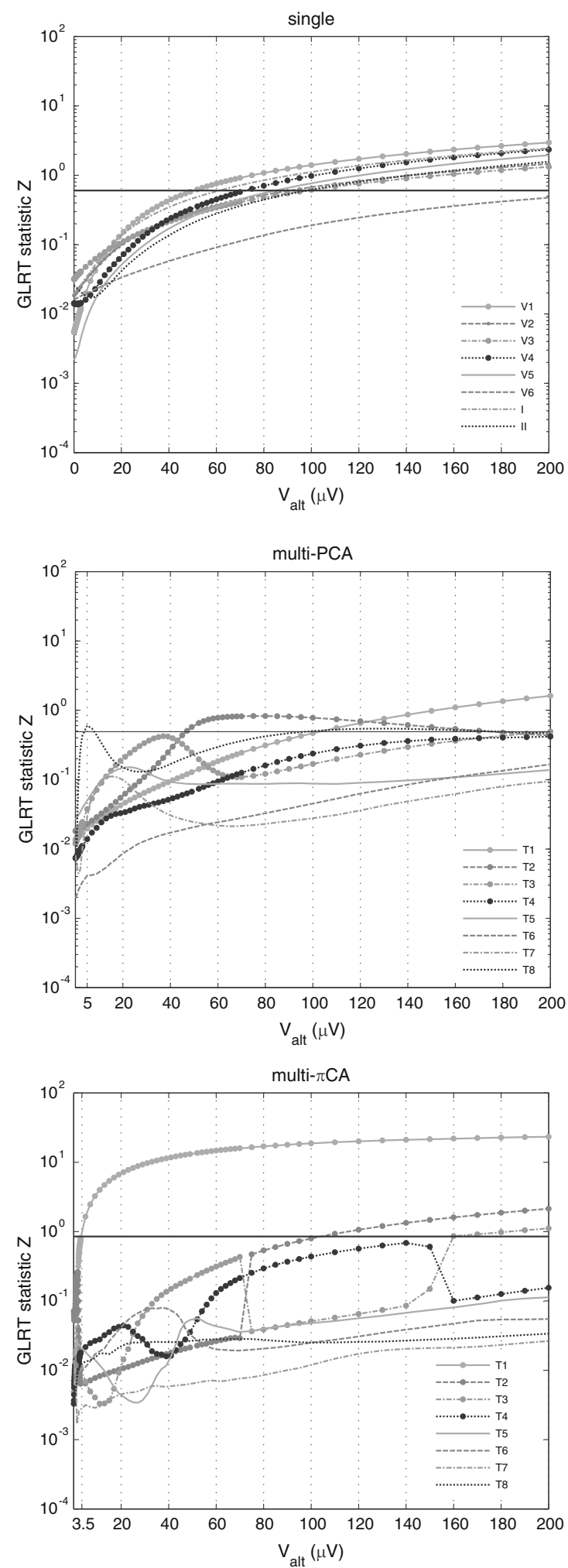

FIGURE 6. Comparison of single (top panel), multi-PCA (middle panel), and multi- $\pi C A$ (bottom panel) schemes applied to the same ECG signal with different magnitudes of TWA Curves represent the values of the detection statistic in the eight leads for each TWA amplitude. Detection is positive when the detection statistic exceeds the threshold, which is indicated with an horizontal straight line $\left(P_{\mathrm{FA}}=\mathbf{0 . 0 1}\right.$ for each scheme). and for each scheme the threshold $\gamma$ was calculated so that it was exceeded only by $1 \%$ of the $Z$ values obtained before the heart rate reached the $H R_{\mathrm{c}}$ (false detections). Then, all records from both groups were processed with the resulting thresholds.

For each detected TWA episode, three parameters were calculated: the maximum amplitude $V_{\max }(\mu V)$, the duration of the episode, and the onset heart rate $H R_{\mathrm{o}}$ (bpm). Average values of amplitude, duration, and onset heart rate were compared between volunteer and ischemic groups with the Mann-Whitney $U$ test, and the percentages of records with TWA of both groups were compared with Fisher's exact test. A $p$-value $<0.05$ was considered significant.

First, $H R_{\mathrm{c}}=110 \mathrm{bpm}$ was chosen to set $\gamma$, and all detected TWA episodes were analyzed (Table 1). The highest sensitivity to TWA was obtained with the multi- $\pi C A$, since it detected TWA in more records than the single scheme both in volunteer (43.9 vs. $28.7 \%$ ) and in ischemic groups (47.1 vs. $28.5 \%$ ). In volunteers, TWA appeared at a lower heart rate than in ischemic patients with both schemes (126 vs. $109 \mathrm{bpm}$ with the multi- $\pi C A$ scheme, 121 vs. $105 \mathrm{bpm}$ with the single scheme).

Then, only TWA episodes detected before $110 \mathrm{bpm}$ (first row in Table 2) and before $100 \mathrm{bpm}$ (second row in Table 2) were analyzed. In both cases, the detection thresholds were recomputed considering cut-off heart rates of $H R_{\mathrm{c}}=110 \mathrm{bpm}$ and $H R_{\mathrm{c}}=100 \mathrm{bpm}$, respectively. With the multi- $\pi C A$ scheme, the percentage of records with TWA was significantly higher in the ischemic group in both cases, whereas this difference was not significant with the single scheme. TWA episodes detected by the two schemes in volunteers had lower amplitude and shorter duration than in the ischemic group, although these differences were not significant.

\section{Signals from the Physionet TWA Database}

The 32 synthetic signals from this database were processed with multi- $\pi C A$, multi-PCA, and single schemes, using an analysis window of 32 beats and the detection thresholds computed in section "Real Signals with Added TWA" under "Results."

In the PhysioNet/Computers in Cardiology Challenge 2008, different methods were proposed for TWA analysis. Each method's TWA estimates were used to develop a ranking of the records in order of TWA amplitude, and then the rankings from different methods were compared using the Kendall rank correlation coefficient. Following this approach, we sorted the records according to the TWA amplitude estimated by each scheme and compared each resulting ranking with the correct ranking that is obtained by sorting the 
TABLE 1. Results of TWA analysis in stress test data, considering all episodes regardless of when they are detected.

\begin{tabular}{|c|c|c|c|c|}
\hline & \multicolumn{2}{|c|}{ Multi- $\pi \mathrm{CA}$ scheme } & \multicolumn{2}{|c|}{ Single-lead scheme } \\
\hline & Volunteer & Ischemic & Volunteer & Ischemic \\
\hline$\%$ records with TWA & $43.9^{a}$ & $47.1^{\mathrm{b}}$ & $28.7^{\mathrm{a}}$ & $28.5^{\mathrm{b}}$ \\
\hline$V_{\max }(\mu V)$ & $125 \pm 143$ & $123 \pm 163$ & $133 \pm 133$ & $135 \pm 146$ \\
\hline Duration (s) & $33 \pm 39$ & $45 \pm 44$ & $29 \pm 24$ & $51 \pm 39$ \\
\hline$H R_{\mathrm{o}}(\mathrm{bpm})$ & $126 \pm 30^{c}$ & $109 \pm 18^{c}$ & $121 \pm 30^{c}$ & $105 \pm 20^{c}$ \\
\hline Number of episodes & 41 & 42 & 26 & 22 \\
\hline
\end{tabular}

$P_{\mathrm{FA}}=0.01$ for the two schemes. Data are expressed as mean \pm one standard deviation).

${ }^{\mathrm{a}, \mathrm{b}}$ Significant difference between multilead and single-lead schemes.

'Significant difference between volunteer and ischemic groups.

TABLE 2. Results of number of records with TWA in stress test data, calculated considering the episodes detected before heart rate reaches $100 \mathrm{bpm}$ (first panel) and $110 \mathrm{bpm}$ (second panel).

\begin{tabular}{|c|c|c|c|c|}
\hline & \multicolumn{2}{|c|}{ Multi- $\pi \mathrm{CA}$ scheme } & \multicolumn{2}{|c|}{ Single-lead scheme } \\
\hline & Volunteer & Ischemic & Volunteer & Ischemic \\
\hline \multicolumn{5}{|l|}{$H R_{\mathrm{c}}<100 \mathrm{bpm}$} \\
\hline$\%$ records with TWA & $7.5^{\mathrm{a}}$ & $24.2^{\mathrm{a}}$ & 7.5 & 14.2 \\
\hline$V_{\max }(\mu \mathrm{V})$ & $89 \pm 87$ & $113 \pm 143$ & $40 \pm 20$ & $128 \pm 130$ \\
\hline Duration (s) & $42 \pm 43$ & $63 \pm 47$ & $25 \pm 17$ & $65 \pm 46$ \\
\hline Number of episodes & 9 & 21 & 6 & 10 \\
\hline \multicolumn{5}{|l|}{$H R_{\mathrm{c}}<110 \mathrm{bpm}$} \\
\hline$\%$ records with TWA & $16.6^{\mathrm{a}}$ & $37.1^{\mathrm{a}}$ & 13.6 & 21.4 \\
\hline$V_{\max }(\mu \mathrm{V})$ & $105 \pm 107$ & $126 \pm 169$ & $72 \pm 55$ & $113 \pm 55$ \\
\hline Duration (s) & $32 \pm 40$ & $49 \pm 39$ & $31 \pm 32$ & $53 \pm 41$ \\
\hline Number of episodes & 13 & 27 & 11 & 15 \\
\hline
\end{tabular}

$P_{\mathrm{FA}}=0.01$ for the two schemes.

aSignificant differences in the number of records with TWA in volunteer and ischemic groups.

records according to its true TWA content. The Kendall rank correlation coefficients between the estimated rankings and the correct ranking were 0.685 for the single scheme, 0.750 for the multi-PCA scheme, and 0.766 for the multi- $\pi C A$ scheme.

\section{DISCUSSION}

The multi- $\pi C A$ scheme presents the best detection performance among the compared schemes, both in synthetic and real signals (Figs. 3 and 5). This scheme exploits the fact that ECG signals do not usually contain any component of cardiac origin at the frequency of $0.5 \mathrm{cpb}$ other than TWA. If baseline wander and other low-frequency artifacts were perfectly removed, the only component projected into the first transformed lead by the $\pi \mathrm{CA}$ transformation would be the alternans. In practice, the signal may still contain residual baseline and noise after preprocessing. In that case, the transformation does not isolate TWA in the first transformed lead, but it still makes TWA stand out over noise, increasing the ANR in that lead. This explains why TWA with an amplitude as low as $5 \mu \mathrm{V}$ becomes detectable in real signals (Fig. 5), and can be clearly observed in the last panel of Fig. 6, where the detection statistic, which represents an indirect measure of the ANR, exceeds the threshold at $V_{\text {alt }}=$ $3.5 \mu \mathrm{V}$.

Unlike $\pi \mathrm{CA}$, PCA does not necessarily project the TWA component into the first transformed lead. PCA separates signal components according to their variance; therefore, PCA projects the component with the highest power into the first transformed lead. That component can be the noise if TWA power is lower than noise power. This effect can be observed in the evolution of the GLRT statistic $Z$ with PCA (middle panel in Fig. 6). In that example, when TWA is low $\left(V_{\text {alt }}=5 \mu \mathrm{V}\right)$, PCA concentrates the noise in the highest transformed leads (T1-T3), removing it from the lowest lead (T8). Therefore, in T8, the ANR rises, the statistic $Z$ passes the detection threshold, and TWA is detected. As $V_{\text {alt }}$ increases, TWA and noise power become similar and PCA cannot separate them, so the ANR in the transformed leads does not increase and TWA is not detected. Then, when $V_{\text {alt }}$ is high enough, 
TWA power starts to be higher than noise power, and PCA progressively projects TWA into higher leads, making them detectable first in T2 and finally in T1. This "positive-negative-positive" detection pattern was consistently found in real signals with added TWA, and is the cause of the "valley" in the $P_{\mathrm{D}}$ curve of the multi-PCA scheme (Fig. 5). This pattern was not found in the synthetic signals of the first dataset, where three leads do not seem to be rich enough in information for PCA to produce such a clear separation of TWA and noise.

The main benefits of the multi- $\pi C A$ scheme can also be expected when it is combined with singlelead techniques other than the LLR method. The improvement in the detection power is due to the signal transformation stage, and does not depend on the actual detection technique, so similar detection gains are obtained when the multilead scheme is combined with LLR and SM techniques. ${ }^{16}$ Furthermore, the multi- $\pi C A$ scheme can also simplify the detection rules of existing methods such as SM, which require a complex set of lead-specific conditions to determine the presence of TWA in an ECG. ${ }^{2}$ The fact that $\pi \mathrm{CA}$ always projects TWA into the first transformed lead (T 1$)$ means that the detection rule needs to be applied only to that lead, making a simpler and more powerful detection scheme.

In addition to a high sensitivity to TWA, an accurate estimation of the TWA amplitude is also an important requisite of the analysis methods, since quantitative evaluation of TWA has been shown to improve risk assessment. ${ }^{8,9,15}$ Results in section "Synthetic Signals" indicate that the multi- $\pi C A$ scheme improves the accuracy of the amplitude estimation (Fig. 4). For high $V_{\text {alt }}$ levels, the bias of the multi- $\pi C A$ estimation is slightly higher than the bias of the singlelead estimation due to the truncation carried out in the reconstruction stage - as only a subset of transformed leads is used to reconstruct the signal, the reconstructed TWA lacks the content of the truncated leads, which may still contain a small alternant component. However, the variance of the multi- $\pi C A$ estimation is lower than the variance of the single-lead estimation, especially at low amplitudes, so the total mean square error is lower than with the single scheme. This effect is also observed in results of section "Signals from the Physionet TWA Database," where the use of the multi$\pi C A$ scheme provides the highest correlation with the correct ranking of records.

Results on stress test signals confirm that the higher sensitivity to TWA of the multi- $\pi C A$ scheme can also be expected in a real clinical dataset. Furthermore, this improvement allows the differentiation of volunteers and ischemic patients according to TWA episodes, which indicates the potential clinical utility of the proposed method. However, cut-off values of heart rate, amplitude, or duration of TWA to predict cardiovascular events could not be determined in the study population because the follow-up information in terms of arrhythmic events or all-cause mortality was not available.

\section{CONCLUSIONS}

The multilead analysis scheme based on $\pi \mathrm{CA}$ is a more robust and sensitive tool for TWA analysis than existing single-lead techniques. Comparison in different scenarios - from synthetic signals to clinical recordsshows that it provides a better detection power than a previously proposed multilead scheme based on PCA, and than the conventional single-lead approach. Furthermore, the proposed scheme provides a method to differentiate between healthy volunteers and patients with ischemic disease according to TWA detections.

\section{ACKNOWLEDGMENTS}

This work was supported by CIBER de Bioingeniería, Biomateriales y Nanomedicina through Instituto de Salud Carlos III and Fondo Europeo de Desarrollo Regional, by Project TEC-2007-68076-C0202 from CICYT, and by Grupo Consolidado GTC from DGA (Spain).

\section{REFERENCES}

${ }^{1}$ Bailón, R., J. Mateo, S. Olmos, P. Serrano, J. García, A. del Río, I. J. Ferreira, and P. Laguna. Coronary artery disease diagnosis based on exercise electrocardiogram indexes from repolarisation, depolarisation and heart rate variability. Med. Biol. Eng. Comput. 41(5):561-571, 2003.

${ }^{2}$ Bloomfield, D. M., S. H. Hohnloser, and R. J. Cohen. Interpretation and classification of microvolt $\mathrm{T}$ wave alternans tests. J. Cardiovasc. Electrophysiol. 13(5):502512, 2002.

${ }^{3}$ Chow, T., D. J. Kereiakes, C. Bartone, T. Booth, E. J. Schloss, T. Waller, E. S. Chung, S. Menon, B. K. Nallamothu, and P. S. Chan. Prognostic utility of microvolt $\mathrm{T}$-wave alternans in risk stratification of patients with ischemic cardiomyopathy. J. Am. Coll. Cardiol. 47(9): 1820-1827, 2006.

${ }^{4}$ Clifford, G. D., F. Azuaje, and P. McSharry. Advanced Methods and Tools for ECG Data Analysis. Norwood, MA: Artech House, Inc., 2006.

${ }^{5}$ Clifford, G. D., S. Nemati, and R. Sameni. An artificial multi-channel model for generating abnormal electrocardiographic rhythms. Physiol. Meas. 31(5):595-609, 2010.

${ }^{6}$ Costantini, O., S. H. Hohnloser, M. M. Kirk, B. B. Lerman, J. H. Baker, B. Sethuraman, M. M. Dettmer, and D. S. Rosenbaum; ABCD Trial Investigators. The ABCD (Alternans Before Cardioverter Defibrillator) trial: Strategies using $\mathrm{T}$-wave alternans to improve efficiency of sudden 
cardiac death prevention. J. Am. Coll. Cardiol. 53(6):471479, 2009.

${ }^{7}$ Cutler, M. J., and D. S. Rosenbaum. Explaining the clinical manifestations of $\mathrm{T}$ wave alternans in patients at risk for sudden cardiac death. Heart Rhythm 6(3 Suppl 1):S22S28, 2009.

${ }^{8}$ de Vilhena Garcia, E., N. Samesima, H. G. P. Filho, C. M. Quadros, L. T. C. da Silva, M. M. Filho, M. L. Z. Hannouche, W. Mathias, and C. A. Pastore. Comparison of quantitative T-wave alternans profiles of healthy subjects and ICD patients. Ann. Noninvasive Electrocardiol. 14(2):108-118, 2009.

${ }^{9}$ Klingenheben, T., P. Ptaszynski, and S. H. Hohnloser. Quantitative assessment of microvolt T-wave alternans in patients with congestive heart failure. J. Cardiovasc. Electrophysiol. 16(6):620-624, 2005.

${ }^{10}$ Martínez, J. P., R. Almeida, S. Olmos, A. P. Rocha, and P. Laguna. A wavelet-based ECG delineator: Evaluation on standard databases. IEEE Trans. Biomed. Eng. 51(4): 570-581, 2004.

${ }^{11}$ Martínez, J. P., and S. Olmos. Detection of T wave alternans in nonstationary noise: A GLRT approach. In: 30th Ann. Conf. Computers in Cardiology. Piscataway, NJ: IEEE Comp. Soc. Press, 2003, pp. 161-164.

${ }^{12}$ Martínez, J. P., and S. Olmos. Methodological principles of $\mathrm{T}$ wave alternans analysis: A unified framework. IEEE Trans. Biomed. Eng. 52:599-613, 2005.

${ }^{13}$ Martínez, J. P., S. Olmos, G. Wagner, and P. Laguna. Characterization of repolarization alternans during ischemia: Time-course and spatial analysis. IEEE Trans. Biomed. Eng. 53:701-711, 2006.

${ }^{14}$ Meyer, C. R., and H. N. Keiser. Electrocardiogram baseline noise estimation and removal using cubic splines and state-space computation techniques. Comput. Biomed. Res. 10(5):459-470, 1977.

${ }^{15}$ Minkkinen, M., M. Kähönen, J. Viik, K. Nikus, T. Lehtimäki, R. Lehtinen, T. Kööbi, V. Turjanmaa, W. Kaiser, R. L. Verrier, and T. Nieminen. Enhanced predictive power of quantitative TWA during routine exercise testing in the Finnish cardiovascular study. J. Cardiovasc. Electrophysiol. 20(4):408-415, 2009.

${ }^{16}$ Monasterio, V., P. Laguna, and J. P. Martínez. Multilead analysis of T-wave alternans in the ECG using principal component analysis. IEEE Trans. Biomed. Eng. 57(7):18801890, 2009.

${ }^{17}$ Moody, G. B. The Physionet/Computers in Cardiology Challenge 2008: T-wave alternans. In: Proc. Computers in Cardiology, pp. 505-508, 2008.

${ }^{18}$ Moody, G. B., W. Muldrow, and R. G. Mark. A noise stress test for arrhythmia detectors. In: Computers in Cardiology, vol. 11. Piscataway, NJ: IEEE Comp. Soc. Press, 1984, pp. 381-384.

${ }^{19}$ Narayan, S. M. T-wave alternans and the susceptibility to ventricular arrhythmias. J. Am. Coll. Cardiol. 47(2):269281, 2006

${ }^{20}$ Nearing, B. D., A. H. Huang, and R. L. Verrier. Dynamic tracking of cardiac vulnerability by complex demodulation of the T wave. Science 252(5004):437-440, 1991.

${ }^{21}$ Nearing, B. D., P. H. Stone, and R. L. Verrier. Frequency response characteristics required for detection of T-wave alternans during ambulatory ECG monitoring. Ann. Noninvasive Electrocardiol. 1:103-112, 1996.

${ }^{22}$ Nearing, B. D., and R. L. Verrier. Modified moving average analysis of $\mathrm{T}$-wave alternans to predict ventricular fibrillation with great accuracy. J. Appl. Physiol. 92(2):541549, 2002.

${ }^{23}$ Rosenbaum, D. S., L. E. Jackson, J. M. Smith, H. Garan, J. N. Ruskin, and R. J. Cohen. Electrical alternans and vulnerability to ventricular arrhythmias. N. Engl. J. Med. 330(4):235-241, 1994.

${ }^{24}$ Sameni, R., C. Jutten, and M. B. Shamsollahi. Multichannel electrocardiogram decomposition using periodic component analysis. IEEE Trans. Biomed. Eng. 55(8):19351940, 2008.

${ }^{25}$ Saul, L. K., and J. B. Allen. Periodic component analysis: An eigenvalue method for representing periodic structure in speech. In: Advances in Neural Information Processing System. Cambridge, MA: MIT Press, 2000, pp. 807-813.

${ }^{26}$ Smith, J. M., E. A. Clancy, C. R. Valeri, J. N. Ruskin, and R. J. Cohen. Electrical alternans and cardiac electrical instability. Circulation 77(1):110-121, 1988.

${ }^{27}$ Turitto, G., E. B. Caref, G. El-Attar, M. Helal, A. Mohamed, R. P. Pedalino, and N. El-Sherif. Optimal target heart rate for exercise-induced T-wave alternans. Ann. Noninvasive Electrocardiol. 6(2):123-128, 2001. 